\title{
SOLAR AND STELLAR CYCLES
}

\author{
GAETANO BELVEDERE \\ Istituto di Astronomia \\ Universita di Catania \\ Italy
}

\begin{abstract}
The increasing observational evidence offered by photometric and spectroscopic data of magnetic cycles in lower main sequence stars, has confirmed the general expectation that the same basic dynamo mechanism operates in the Sun and main sequence in stars with outer convective envelopes.
\end{abstract}

Unfortunately, no clear correlation has been found, up to date, with stellar parameters as mass, rotation rate and age, even if irregular activity and shorter cycle periods seem to be characteristic of stars more massive than the Sun, while hyperactive fast rotating components of binary systems like RS CVn's and BY Dra's show a tendency for cycles as long as several decades.

Although dynamo theory has probably captured the essential physics of the convection-rotation interaction giving rise to stellar magnetic activity, as evidenced, for instance, by the correlation between proxy activity, indicators and the Rossby parameter related to the dynamo number, the reliability of the present theoretical background should be measured by its capacity of interpreting and predicting characteristics and periodicities (or aperiodicities) of stellar cycles. This should be done in the framework of the nonlinear approach, which, in principle can describe multimodal dynamo behaviour with a variety of time scales.

The fundamentals of the theory must be tested, however, in the closest astrophysical laboratory, our Sun. Serious problems to a dynamo mechanism operating in the convection zone have been posed by most recent helioseismological results, which, on the other hand, do not rule out the possibility of dynamo action in the transition layer between the convective and the radiative zones, which is suggested independently by the global solar cycle features. Indeed, assuming the correct sign of helicity in the transition layer, the helioseismological data on the radial gradient of angular velocity support both equatorward propagation of dynamo waves at lower latitudes and poleward propagation at higher latitudes, which is evidenced by different tracers of the solar cycle.

\section{The Principle of Solar-Stellar Connection}

This is an unification principle. It states: (i) that the phenomenology of solar and stellar magnetic activity is essentially the same despite of different strength, topology and time scale of active phenomena occurring on the Sun and distant stars; and (ii) that this phenomenology can be interpreted in terms of the same fundamental mechanism, the dynamo mechanism, operating in stellar convection zones, although differences in the dynamo operation mode are expected,depending on stellar mass, rotation rate and age, as well as on the dynamo number, a parameter which characterizes the 
strength of dynamo action - the last point being clearly evidenced by the non-linear analysis of dynamo equations.

Hence, the complementarity of solar and stellar observations to highlight the basic phenomena and mechanisms of stellar activity:

- in a way, solar data give the possibility of detailed study of activity features and phenomena in the closest astrophysical laboratory (the Rosetta Stone of Astrophysics), provide a guide-line to explore stellar activity on the basis of what we leam from the Sun, and allow to test models of stellar activity "at home"

- on the other side, stellar observations do offer a large sample of phenomenology on a multiplicity of astrophysical situations and time scales, suggesting a more general scenario and widening out our ideas about the operation modes of stellar activity.

Unity in multiplicity is therefore the basic concept of the solar - stellar connection: unity as for the mechanism, multiplicity as for its operation modes depending on stellar parameters.

But, warning! Some caution is necessary when making use of the analogy to the Sun to understand phenomena in stars of different activity level. For instance :

- extrapolating from slow rotators (hypoactive stars) to fast rotators (hyperactive stars) may be seriously dangerous

- the dynamo operation mode may be different in different activity level stars, even as to location (the convection zone(c.z.) or the boundary layer at the base of the c.z. or even the stellar core) and driving mechanism itself (radial or latitudinal angular velocity gradient in the stellar interior)

- significant differences in the activity signatures of very similar stars are expected when the analysis is made in the non - linear magnetohydrodynamic regime (Weiss et al. 1984, Belvedere et al. 1989, Belvedere and Proctor 1989). This is confirmed on the observational side (Rodono 1987).

Therefore, dynamo theory must be tested both on the Sun and distant stars and is reliability measured by its capacity of interpreting and predicting different activity signatures in different stars. In this context, it appears to be of outstanding relevance to confront theory and observations as to periodicities or aperiodicites in the time evolution of activity, with particular reference to stellar cycles.

\section{Some Relevant Features of the Solar Cycle}

As is known, proxy records of solar activity, as C's fluctuations in tree rings or Be's in polar ice caps (Stuiver and Quay 1980) allow us to explore the activity of the Sun back to about nine millenia ago. Note that the layered Precambrian sediments found in South Australia, which were previously interpreted as an evidence for solar cyclic activity some 700 million years ago, were due to tidal processes (Williams 1989; Weiss 1989).

Human sporadic sunspot observations date back to two millenia ago, but systematic spot counts only to mid - seventeenth century.

It has clearly been stated on the basis of spot counts (Wolf number) that the mean periodicity of the solar cycle is 11.2 years, with range from 8 to 15 years. More correctly, one has to take the double (22y) as the real solar cycle period, this being the time interval between two successive appearances of the same magnetic field polarity at the solar poles. Also long - term periodicities in the sunspot counts have been evidenced: the $80-90$ y Gleissberg cycle and the 190 y Grand Cycle. For details on the solar cycle features see e.g. Stix (1981). 
Curiously, it is not the modulation of activity which is more relevant to understand the solar cycle (and the underlying dynamo mechanism) but the nearly absence of activity at certain epoches, the so called Grand Minima (Eddy 1976, 1983): Oort's Grand Minimum (1010-1050 A.D.), Wolf's (1280-1340), Spörer's (1420-1530) and Maunder's (1645-1715).

Note also that in the dendrochronologic record episodic cycle suppression occurs roughly at 250 year intervals and lasts about one third of this time interval (Damon 1977). This indicates that the solar cycle is really intermittent or, in other words, it exhibits chaotic behaviour with different time scales and episodes of reduced or zero activity, namely a real aperiodicity rather than a quasi - periodicity (Williams 1981, Wallenhorst 1982). To this regard one may question how significant using the term "solar cycle" is, at least referring to suitably extended time intervals.

However, interpreting the solar cycle anomalies in terms of stochastic behaviour instead of deterministic chaos cannot be excluded. The matter is still controversial, but, to this regard, the relation between mean magnetic field and stochastic turbulent convection may be relevant (Hoyng 1987, 1988).

Now, what about stellar cycles? Before entering this problem, let us examine the indicators of magnetic activity in stars and their relations to the basic stellar parameters as mass, rotation rate and age.

\section{Stellar Activity Indicators}

\subsection{MAGNETIC FIELD MEASUREMENTS}

Robinson's (1980) method allows to measure non-averaged magnetic field strengths and filling factors by means of comparing magnetic sensitive and insensitive lines. This avoids cancelling out of opposite polarities, which occurs in conventional Zeeman polarization measurements. Results for 29 G-K stars (with 19 detections) have been reviewed by Marcy (1984), indicating magnetic field intensities $B$ as high as $1500 \div 2000$ Gauss, filling factors $f$ in the range $0.3 \div 0.8(>>$ than the solar value $\sim 0.01$ ) and a correlation between magnetic flux density, effective temperature and rotational velocity of the type $\Phi \approx T_{e f f}^{-2.8} V_{\text {rot }}^{0.55}$. Note that the Sun does not fit in with this relationship! Gray (1984) found a similar trend. Saar and Linsky (1986), after measuring 20 main sequence stars, found that the magnetic field intensity B correlates with the surface pressure (related to $T_{e f f}$ ), as suggested by theoretical arguments on flux tube equilibrium, but does not with the rotational velocity: it is indeed the magnetic flux $\Phi \approx f B$ which correlates with $V_{\text {rot }}$. This is an important observational result, as it shows: (i) that the relevant quantity to be related to the rate of rotation in dynamo theories is the magnetic flux; and (ii) that for similar stars $B$ and $f$ may be inversely proportional. More recently (Saar 1989), it has been shown that $\Phi$ is proportional to the product $\tau_{c} \omega$, where $\tau_{c} \mathrm{~s}$ the convective turnover time (see later) and $\omega$ the angular velocity of rotation, and declines exponentially with stellar age.

Future improvement of the observational techniques will check the previous results about magnetic field strengths and filling factors, which are essential data for testing dynamo theory. To this regard, we look with interest at the recent Zeeman - Doppler Imaging Method (Donati, Semel and Praderie,1988; Semel 1989) which can give both intensity and cartography of magnetic fields on stellar surfaces, by measuring both I and V Stokes parameters. In principle, this is an extension of the Doppler Imaging Method (Vogt 1983; Vogt et al. 1987), which allows to reveal spotted regions on stars. 


\subsection{PHOTOMETRIC VARIABILITY}

This evidences the presence of dark spots on stellar surfaces and can in principle give us information on periodicities or aperiodicities in stellar activity as well as on differential rotation of stellar envelopes (large and short scale variation in time of stellar luminosity). We do not go into details here, referring the reader to Byme and Rodono (1983), Baliunas and Vaughan (1985) and Rodono (1987). We just outline that three levels of activity have been evidenced

- I level (Hyperactivity)

This is characteristic of the fast rotating RS Canum Venaticorum and BY Draconis type stars, with luminosity variation amplitude $A$ up to $0.6 \div 0.7 \mathrm{mag}$, filling factor $\mathrm{f}$ up to several tenths $(>50 \%)$ and time scale (cycle period?) of the order of $50+60 \mathrm{y}$

- II level (Mid - activity)

This refers essentially to young and fast rotating stars in Pleiades and Hyades, with A 0.1 mag, $f \sim 0.1$

- III level (Hypoactivity)

This is the class to which most stars, generally slow rotators, belong, including our Sun, with $\mathrm{A} \sim 0.01 \div 0.05, \mathrm{f} \sim 0.01$ and time scale (cycle period) $\sim 10 \mathrm{y}$.

Before entering the next subsection, we point out the important fact that both photospheric flux modulation amplitude and chromospheric emission flux do increase from level III to level I, this giving consistency to our global understanding of stellar activity.

\subsection{SPECTROSCOPIC VARIABILITY}

We refer to $\mathrm{Ca}_{\Pi} \mathrm{H}, \mathrm{K}$ and $\mathrm{Mg}_{\mathrm{II}} \mathrm{h}, \mathrm{k}$ line emission cores, which are a powerful tool to investigate stellar activity, as clearly shown since the pioneeristic work by Wilson (1978). For all details about the method and observational results we address the reader to Catalano (1984), Baliunas and Vaughan (1985), Hartmann and Noyes (1987), Baliunas et al. (1989), and references therein. Chromospheric fluxes (emission cores), originating from active regions are very good proxy indicators of stellar activity, being correlated to the magnetic field strength and filling factor (Skumanich et al. 1975).

The flux modulation in time allows to measure both rotation rates (of course, independent of $\mathrm{V}$ sin i) and activity time-scales, including cycle periods. The reliance on proxy indicators of stellar activity derived from the difficulty of directly measuring the magnetic field topology and intensity.

However, due to the progress in the techniques of investigation, calibrating the emission flux in terms of the magnetic flux density $\Phi=f B$ is now possible. For the Sun we have (Schrijver et al. 1989):

$$
\log \Delta F_{H, K}=0.6 \log (f B)+4.8
$$

and, for a sample of active stars (Saar and Schrijver, 1987; Schrijver, 1987) :

$$
\Delta F_{H, K} \sim(f B)^{0.62 \pm 0.14} \text { for }(\mathrm{fB})<300 \text { Gauss }
$$

where $\Delta F_{H, K}$ is the "excess" emission flux, defined in the next subsection.

The substantial agreement between the relationships found for the Sun and the sample of stars is a further evidence of the correctness of the solar-stellar connection principle. 


\subsection{THE DEPENDENCE OF CHROMOSPHERIC ACTIVITY ON STELLAR PARAMETERS}

Different proxy indicators of chromospheric activity have been used in recent years :

(i) the emission flux $F_{H, K}$

(ii) the ratio $R_{H, K}$ of the emission flux to the bolometric flux

(iii) the "excess" emission flux $\Delta F_{H, K}$, namely the difference between the total flux and the "basal" flux, the latter representing the contribution of the quiet chromosphere

(iv) the emission luminosity $L_{H, K}$

Noyes et al. (1984a) found a correlation between $\log R_{H, K}$ and $\log P_{\text {rot }}$, where $P_{\text {rot }}$ is the rotational period.

However, they noted that the scatter is minimized when correlating $\log R_{H, K}$ and $\log P_{\text {rot }} / \tau_{c}$, where $R_{0}=P_{\text {rot }} / \tau_{c}$ is the Rossby parameter and $\tau_{c}$ is the convective turnover time, namely the ratio of the scale height at the base of the convection zone (c.z.) to the characteristic convective velocity in the deep c.z. This is the order of magnitude of the time necessary for an element of fluid to describe a closed path in a rotating convection zone. Note that $\tau_{c}$ is stellar mass dependent, as the structure and dynamics of the convective zone of a star depend on its mass (or colour index, (B-V)).

Therefore the Rossby parameter is a function of both mass and rotation rate. Note that this parameter is a "hybrid", as $P_{\text {rot }}$ is an observed quantity and $\tau_{c}$ a theoretical one, computed by Gilman (1980).

But the important point is the following. Increasing $\mathbf{R}_{\mathbf{0}}$ means increasing the rotational period and/or decreasing $\tau_{c}$ i.e. the thickness of the stellar c.z. In other words, $R_{0}$ increases for slower rotators and/or earlier spectral types, and the observed correlation between $R_{H, K}$ and $\mathrm{R}_{0}$ shows that activity declines with increasing $R_{0}$. This is consistent with dynamo theory : in fact $R_{0} \sim 1 / \sqrt{D}$, (see e.g. Belvedere 1985), where $\mathrm{D}$ is the dynamo number which parametrizes the effect of the interaction of convection and rotation giving rise to dynamo action, the strength of which increases with increasing $\mathrm{D}$. Therefore, the larger is $\mathrm{R}_{0}$, the weaker is dynamo action, in agreement with the observational evidence. Note that this argument was already in Durney and Latour (1978) and Belvedere et al. (1980a).

Inverting the argument one can conclude that stellar activity gets stronger in faster rotators and later spectral types.

Zwaan (1986), Rutten and Schrijver (1986), using basically the same data, but $\Delta F_{H, K}$ as the proxy indicator, did not find a unique correlation between chromospheric activity, rotation rate and mass. They indeed obtained a family of curves in the plane $\left(\log \Delta_{H, K}, \log P_{\text {rot }}\right)$, with slopes depending on (B-V). A detailed discussion on this discrepancy can be found in Hartmann and Noyes (1987).

Moreover, Marilli et al. (1986), using another proxy indicator, the chromospheric emission luminosity, found a correlation such as :

$$
L_{H, K} \sim e^{-\alpha P_{\text {rot }}}
$$

where the interesting fact is that a, derived by observations nearly coincides with $1 / \tau_{c}$, computed by Gilman (1980). 
At the present, all the authors here mentioned claim to have used the best chromospheric activity physical indicator. We do not enter upon this subject here, but stress the fact that, independently of the particular indexes selected as tracers (spots, active chromospheric regions) or proxy indicators $\left(R_{H, K}, \Delta F_{H, K}, L_{H, K}\right)$ the observational evidence shows that activity increases with increasing rotation rate and c.z. thickness. This is also confirmed by direct magnetic field strength and filling factor measurements, and is in agreement with the predictions of dynamo theory.

\section{Stellar Activity Cycles : Observations and Theory}

In the last twenty years, a great amount of data on chromospheric variability of active stars have been collected on a sample of about 100 objects (see Baliunas and Vaughan 1985, Baliunas 1986, Baliunas et al. 1989).

The results indicate that $15 \%$ of the observed stars show no variability, $25 \%$ show chaotic or erratic time behaviour and the remaining $60 \%$ show cyclic time behaviour, with periods of the order of $10 \mathrm{y}$, mostly ranging from 5 to 15 years. Also most cycle characteristics are solar - like, as to smoothness and shape. Further a Maunder minimum - like behaviour has been detected on the star HD 10700.

Cycle periods $<5$ y have been observed only on stars with mass $M>M_{0}$, this having some relevance as for the comparison between theory and observations, as pointed out later. However, and this is a very important point, no clear correlation has been found between the cycle period and stellar parameters as rotational period, Rossby parameter, mass (or spectral type), chromospheric activity strength (i.e. magnetic flux density) and age.

Perhaps the observational time span is too short (?) and the sample too limited (?), so that we have to wait for new observations prior to drawing any conclusions. If the present lack of correlation is confirmed, dynamo theory will confront a new challenge, since up-to-date predictions are few and controversial, and disagree with the observational evidence.

Let us have a look at the theoretical investigation on stellar cycles and their relation to stellar parameters.

According to Belvedere et al. (1980c), the cycle period should increase with (B-V), this result being obtained in the case of marginal dynamo instability for which the dynamo wave period is of the order of the diffusion time in the stellar convection zone.

This result is just the opposite of what has been found by Dumey and Robinson (1982), according to whom the period should decrease with (B-V), assuming the dynamo wave period to be of the order of the magnetic field amplification time in the convective layers.

As to the $P_{\text {ror }}$-dependence, both models predict $P_{\text {cycle }} \sim P_{\text {rot }}$ (see e.g. Belvedere 1985). More recently Noyes et al.(1984b) found the empirical relationship $P_{\text {cycle }} \sim\left(P_{\text {rot }} / \tau_{c}\right)^{1.25}$ and discussed it in the framework of a simple nonlinear dynamo model. However, the number of stars was too limited (12 objects), so that their result was not confirmed once the sample was extended to all the measured stars (Baliunas and Vaughan 1985, Baliunas et al. 1989).

Therefore these models not only disagree with each other, but, further, have scarce or no observational support, since no cycle dependence on the spectral type or the rotational period has been found up to now. 
Perhaps, however, the limited evidence of short cycle periods in late F-type stars and long cycle periods in RS CVn and BY Dra gives some slight support to Belvedere et al. (1980c), as well as the $P_{\text {rot }}$ independence of $P_{\text {cycle }}$ is consistent with the theoretical result of Kleeorin et al. (1983) for the case of rapid rotators.

In the light of these non-encouraging theoretical predictions on stellar cycles, a question naturally arises: is dynamo theory well founded and at least partially reliable, even if same caution is undoubtedly necessary? To explore the implications of this question and try to answer it, let us come back home, to the solar dynamo.

\section{The Present Status of Solar Dynamo Theory}

As is known, dynamo theory attempts to explain the generation and evolution of cosmical magnetic fields in terms of induction effects in rotating conducting fluid masses. For the development of the main concepts and exhaustive detailed information, we address the reader to the books of Moffatt (1978), Parker (1979), Krause and Rädler (1980), and to the reviews of Stix (1981), Schüssler (1983), Belvedere (1985).

Rather a lot of solar dynamo models have been dished up in recent and less recent years, most based on the original Parker's (1955) formulation in terms of the so called $\alpha-\omega$ mechanism: toroidal magnetic fields are generated from poloïdal ones by differential rotation ( $\omega$-effect), while cyclonic turbulence ( $\alpha$-effect) regenerates poloïdal fields from toroïdal ones (e.g. Steenbeck and Krause 1969 a,b; Yoshimura 1975, 1983; Belvedere et al. 1980b, 1980c, 1987, 1989; Schüssler 1984; Gilman 1983, 1986; Gilman and Miller 1981, Gilman et al. 1989; Durney and Robinson 1982, Ruzmaikin 1984; Dumey 1988, Brandenburg et al. 1988, 1989, Schmitt and Schüssler 1989).

Dynamo models may be separated into two classes: cinematic, linear dynamos and hydromagnetic, non-linear dynamos. In the latter the back-reaction of the magnetic field on the velocity field is taken into account, and the whole system of the magnetohydrodynamic equations is solved simultaneously, assuring internal consistency.

Although linear models seem to have captured the essential physics of the convection-rotation interaction giving rise to dynamo action, as is shown by their capacity of reproducing the solar cycle, there is no doubt, however, that present and future research has to be carried out in the framework of the nonlinear approach, which in principle allows to describe a multiplicity of dynamo operation modes and to predict magnetic field strengths.

Here we do not enter upon the subject of solar activity models, but outline some recent basic problems and developments of solar dynamo.

\subsection{THELOCATIONAND WORKINGMECHANSMOF DYNAMOIN THELGHTOF HELOSEISMOLOGY}

The location of dynamo action has vigorously been debated in recent years. Three possible locations have been suggested in modelling solar dynamo:

(i) the whole convection zone

(ii) the base of the convection zone (1st scale height)

(iii) the boundary (overshoot) layer between the convective and the radiative zones.

The last possibility seems to be the most realistic on the basis of the following argument.Spatial separation of the $\omega$-effect and the $\alpha$-effect is not plausible in so far as it would imply problems of 
upward and downward field transport in a turbulent medium. On the other hand, since stability of magnetic flux tubes against magnetic buoyancy suggests that the $\omega$-effect is located deep in the convection zone or better in the boundary layer (Parker 1979, Spiegel and Weiss 1980, Spruit and Van Ballegooijen 1982), also the $\alpha$-effect is expected to operate mainly at deep levels. This is also supported by the argument that the $\alpha$-effect on flux tubes rapidly rising to the surface would be ineffective in the top half of the convection zone. (Golub et al. 1981).

The location of dynamo in the boundary layer is indirectly supported by the helioseismic results. The most recent helioseismological data (Duvall et al. 1986, Brown and Morrow 1987; Christensen - Dalsgaard and Shou 1988; Harvey 1988; Libbrecht 1988, 1989; Dziembowski et al. 1989; Brown et al. 1989) seem to agree as to the following (provisional) scenario:

(i) the surface angular velocity $\omega\left(R_{0}, \theta\right)$ persists throughout the convection zone $\left(1 \mathrm{R}_{\Theta} \rightarrow 0.7 \mathrm{R}_{\odot}\right)$ : i.e. $(\mathrm{d} \omega / \mathrm{dr})_{\text {c.z. }} \sim 0$ or slightly $>0$

(ii) beneath the convection zone $\left(0.7 \mathrm{R}_{\mathcal{O}} \rightarrow 0.65 \mathrm{R}_{\mathcal{O}}\right)$, rigid rotation dominates with $\omega_{0}=2.710^{-6} \mathrm{rad} / \mathrm{s}$ which is the surface value at latitude $\sim 37^{\circ}$. All this implies that the equatorial and polar rotation rates do converge to the intermediate value $\omega_{0}$ below the convection zone.

Let us see what the implications to solar dynamo are :

(i) radial shear driven dynamo, operating in the convection zone might be unrealistic

(ii) radial shear driven dynamo operating in the boundary layer is still supported by helioseismological data. The argument is the following (with reference to the northern hemisphere).

Since $\alpha<0$ in the boundary layer (Yoshimura 1975, Gilman 1983, Glatzmaier 1984, 1985a,b, Gilman et al. 1989, Parker 1989), and, interpolating the helioseismological results in the boundary layer:

$$
\begin{aligned}
& \frac{d \omega}{d r}<0 \text { at high latitudes }\left(\geqslant 37^{\circ}\right) \\
& \frac{d \omega}{d r}>0 \text { at low latitudes }\left(\leqslant 37^{\circ}\right)
\end{aligned}
$$

we get poleward migration of the toroidal field at high latitudes $\left(\alpha \frac{d \omega}{d r}>0\right.$ ) and equatorward migration at low latitudes $\left(\alpha \frac{d \omega}{d r}<0\right.$ ), in agreement with the observational evidence shown by different tracers of the solar cycle (polar faculae and prominences on the one side, spots and faculae on the other side).

This means that dynamo can still work and reproduce the observations if is located in the boundary layer.

At present, however, all must be taken with great caution! Indeed :

- the helioseismic inversion data are not conclusive. For instance, noise may mask the effect of rotation (Brown et al. 1989).

- discrepancies do exist between different sets of data (see e.g. Rhodes et al. 1987, 1988)

- convection zone dynamo models which may work, have still been proposed: magnetostrophic wave dynamo (Schmitt 1987) cylindrical isorotation dynamo (Durney 1989a,b) latitudinal shear driven dynamo (Parker 1989). 


\section{Concluding Remark}

Dynamo theory has some obscure and bright aspects. The next decade will probably decide on its validity.

We refer above all to:

- the impact of helio- and asteroseismology

- the impact of the recently observed resonant structure of the magnetic pattern of the solar cycle (Stenflo and Vogel 1986, Stenflo 1988),characterized by different stability of odd and even parity modes with respect to the equator and different $l$ dependences of the mode frequency and then, of the associated sinusoidal period, for odd and even modes ( $l$ is the spherical harmonic degree)

- the impact of the present no - correlation between stellar cycle periods and stellar parameters, or, in the future, of any (expected?) correlation found when a larger sample of stellar data is available.

The one we are living is probably a period of transition, in which a pause of meditation and reflection is perhaps necessary.

\section{Acknowledgements}

I want to express my friendly thanks to many colleagues for kindly sending me their recent work: S.L.Baliunas, A.Brandenburg, T.M.Brown, B.R.Durney, P.Foukal, P.A.Gilman, B.M.Haisch, P.Hoyng, R.W.Noyes, E.N.Parker, L.Paternõ, F.Praderie, R.Rosner, R.J.Rutten, M.Schüssler, D.R.Soderblom, H.C.Spruit, J.O.Stenflo, M.Stix, I.Tuominen, G.S.Vaiana, A.A.Van Ballegooijen, H.B.Van der Raay, S.S.Vogt, N.O.Weiss, M.F.Woodard, C.Zwaan.

I sincerely apologize to those ones whose papers I didn't quote, due to the limited extent of this review.

I also enjoyed helpful e-mail and letter discussions with Bernard Durney and Peter Hoyng.

\section{References}

Baliunas, S.L. and Vaughan, A.H.: 1985, Ann. Rev. Astron Astrophys. 23, 379

Baliunas, S.L.: 1986, in Cool Stars, Stellar Systems and the Sun, M.Zeilik and D.Gibson eds., Springer, Berlin, p.3

Baliunas, S.L. Donahue, R.A., Horme, J.H, Noyes,R.W., Porter, A., Gilliland, R.L. Duncan, D.K., Frazer, J. Lanning, H., Misch, A., Mueller, J., Soyumer, D., Vaughan, A.H., Wilson, O.C. and Woodard, L.A.: 1989, Astrophys. J., in press

Belvedere, G.: 1985, Solar Phys. 100, 363

Belvedere, G. and Proctor, M.R.E.: 1989, in Solar Photosphere : Structure, Convection and Magnetic Fields, J.O. Stenflo ed., Kluwer, Dordrecht, in press

Belvedere, G., Paternõ, L. and Stix, M.: 1980a, Astron. Astrophys. 86,40

Belvedere, G., Paterno, L. and Stix, M.: 1980b, Astrophys. 88, 240

Belvedere, G., Patemo, L. and Stix, M.: 1980c Astron. Astrophys. 91 , 328

Belvedere, G., Pidatella, R.M. and Proctor, M.R.E.: 1989, Geophys Astrophys. Fluid Dyn, in press

Belvedere, G., Pidatella R.M. and Stix, M.: 1987, Astron. Astrophys. 177, 183 
Brandenburg, A. and Tuominen, I.: 1988, Adv. Space Res. 8,

Brandenburg, A. Krause, F., Meinel, R.,Moss, D. and Tuominen, I.: 1989

Astron. Astrophys 213, 411

Brown, T.M. and Morrow, C.A.: 1987, Astrophys. J. 314, L21

Brown, T.M. Christensen-Dalsgaard, J.,Dziembowski, W., Goode, P.R., Gough, D.O. and Morrow C.A.: 1989, Astrophys. J. 343, 526

Byme P.B. and Rodonõ, M. (eds.): 1983, Activity in Red-Dwarf Stars, Reidel, Dordrecht.

Catalano, S.: 1984, in Space Research Prospects in Stellar Activity and Variability, A. Mangeney

and F. Praderie eds., Observatoire Meudon, p. 243

Christensen - Dalsgaard, J. and Shou, J.: 1988, in Seismology of the Sun and Sun-like Stars,

E.J. Rolfe ed., ESA-SP 268, p. 55

Damon, P.E.: 1977, in The Solar Output and Its Variation,O.R. White ed., Colorado. Ass.

Univ. Press., Boulder, p. 429

Donati, J.F., Semel, M. and Praderie, F.: 1989, Astron. Astrophys in press

Durney, B.R.: 1988, Astron. Astrophys., 191, 374

Durney, B.R.: 1989a, Astrophys. J, 338, 509

Dumey, B.R.: 1989b, Solar Phys., in press

Dumey, B.R. and Latour. J: 1978, Geophys. Astrophys. Fluid Dun. 9, 241

Dumey, B.R. and Robinson, R.D.: 1982, Astrophys. J. 253, 290

Duvall, T.L., Harvey, J.W. and Pomerantz, M.A.: 1986, Nature 321, 500

Dziembowski, W.A., Goode, P.R. and Libbrecht, K.G. 1989, Astrophys. J. 337, L53

Eddy, J.A.: 1976, Science 286, 1189

Eddy, J.A.: 1983, Solar Phys. 89, 195

Gilman, P.A.: 1980, in Stellar Turbulence, D.F. Gray and J.L.Linkly eds.,

Springer, Berlin, p. 19

Gilman, P.A.: 1983, Astrophys. J.Suppl. 53, 243

Gilman, P.A.: 1986, Geophys. Astrophys. Fluid Dyn. 31, 137

Gilman, P.A. and Miller, J.: 1981, Astrophy. J. Suppl. 46,

Gilman, P.A., Morrow, C.A. and De Luca, E.E.: 1989, Astrophys. J. 338, 528

Glatzmaier, G.A.: 1984, J.Comp. Phys. 55, 461

Glatzmaier, G.A.: 1985a, Geophys. Astrophys. Fluid Dyn. 31, 137

Glatzmaier, G.A.: 1985b, Astrophys. J. 291, 300

Golub, L., Rosner, R., Vaiana, G.S., and Weiss, N.O.: 1981, Astrophys. J. 243, 309

Gray, D.F.: 1984, Astrophys. J. 277, 640

Hartmann, L.W. and Noyes, R.W.: 1987, Ann. Rev. Astron. Astrophys. 25, 271

Harvey, J.W.: 1988, in Seismology of the Sun and Sun-like Stars, E.J. Rolfe ed., ESA-SP 286, p.55

Hoyng, P.: 1987a, Astron. Astrophys. 171, 348

Hoyng, P.: 1987b, Astron. Astrophys. 171, 357

Kleeorin, N.I., Ruzmaikin, A.A. and Sokoloff, D.D.: 1983, Astrophys, Space Sci. 95, 131

Krause, F. and Rä dler, K.H.: 1980, Mean Field Magneto hydrodynamics and Dynamo

Theory, Pergamon Press, Oxford

Libbrecht, K.G.: 1988, in Seismology of the Sun and Sun-like Stars, E.J. Rolf ed.

ESA-SP 286, p.131

Libbrecht, K.G.: 1989, Astrophys. J. 336, 1092

Marcy, G.W.: 1984, Astrophys. J. 276, 786

Marilli, E., Catalano, S. and Trigilio, C.: 1986, Astron. Astrophys. 167, 297 
Moffatt, H.K.: 1978, Magnetic Field Generation in Electrically Conducting Fluids, Cambridge Univ.Press

Noyes, R.W., Hartmann, L.W., Baliunas, S.L., Duncan, D.K. and Vaughan, A.H.: 1984a, Astrophys. J. 279, 763

Noyes, R.W., Weiss N.O. and Vaughan, A.H.: 1984b, Astrophys. J. 287, 769

Parker, E.N.: 1979, Cosmical Magnetic Fields, Clarendon, Oxford

Parker, E.N.: 1987, Solar, Phys. 110, 11

Parker, E.N.: 1989, in Solar and Stellar Flares, B.M.Haisch and M.Rodonõeds., Solar Phys., in press

Rhodes, E.J., Cacciani, A., Woodard, M.F. Tomczyk, S., Korzennik, S. and Ulrich, R.K.: 1987, in The Internal Solar Angular Velocity, B.R. Durney and S. Sofia, eds.

Kluwer, Dordrecht, p. 75

Rhodes, E.J. Cacciani A., Tomczyk, S., Ulrich, R.K. and Woodard, M.F.: 1988, in

Seismology of the Sun and Sun-like Stars, E.J. Rolfe ed., ESA-SP 286 p.73

Robinson, R.D.: 1980, Astrophys. J. 239, 261

Rodonõ, M.: 1987, in Solar and Stellar Physics, E.H. Schröter and M. Schüssler eds., Springer, Berlin, p.39

Rutten, R.G.M. and Schrijver, C.J.: 1986, in Cool Stars, Stellar Systems and the Sun, M.Zeilik and D.Gibson eds., Springer, Berlin, p.120

Ruzmaikin, A.A.: 1984, in Proceed. 4th European Meeting on Solar Phys., T.D. Guyenne and J.J. Hunt eds., ESA SP-220, p.85

Saar, S.H.: 1989, in Solar Photosphere : Structure, Convection and Magnetic Fields, J.O. Stenflo ed., Kluwer, Dordrecht, in press

Saar, S.H. and Linsky, J.L.: 1986, Adv. Space Res. 6, 235

Saar, S.W. and Schrijver, C.J.: 1987, in Cool Stars, Stellar Systems and the Sun, J.L. Linsky and R.E. Stencel eds., Springer, Berlin, p. 38

Schmitt, D.: 1987, Astron. Astrophys. 174, 281

Schmitt, D. and Schüssler, M.: 1989, Astron. Astrophys. submitted

Schrijver, C.J.: 1987, Astron. Astrophys. 172, 111

Schrijver, C.J., Coté, J., Zwaan C. and Saar S.H.: 1989, Astrophys. J. 337, 964

Schüssler, M.: 1983, in Solar and Stellar Magnetic Fields : Origin and Coronal Effects, J.O.Stenflo ed., Reidel, Dordrecht, p. 213

Schüssler, M.: 1984, in Proceed. 4th European Meeting on Solar Physics, T.D. Guyenne and J.J. Hunt eds., ESA SP-220, p. 67

Semel, M.: 1989, Astron. Astrophys., submitted

Skumanich, A., Smythe, C., Frazier, E.N.: 1975, Astrophys., J. 200, 747

Spiegel, E.A. and Weiss, N.O.: 1980, Nature 287, 616

Spruit, H.C. and Van Ballegooijen, A.A.: 1982, Astron. Astrophys. 106, 58

Steenbeck, M. and Krause, F.: 1969, Astron. Nachr. 291, 49

Steenbeck, M. and Krause, F.: 1969, Astron. Nachr. 291, 271

Stenflo J.O.: 1988, Astrophys. Space Sci. 144, 321

Stenflo J.O. and Vogel, M.: 1986, Nature 319, 285

Stix, M.: 1981, Solar Phys. 74, 79

Stuiver, M. and Quay, P.D.: 1980, Science 207, 11

Vogt, S.S.: 1983, in Activity in Red-Dwart Stars, P.B. Byrne and M. Rodonõ eds., Reidel, Dordrecht, p. 137

Vogt, S.S., Penrod, G.D. and Hatzes, A.P.: 1987, Astrophys J. 321, 496

Wallenhorst, S.G.: 1982, Solar Phys. 80, 379 
Weiss, N.O.: 1989, in Accretion Disks and Magnetic Fields in Astrophysics, G.Belvedere ed., Kluwer, Dordrecht, p.11

Weiss, N.O., Cattaneo, F. and Jones, C.A.: 1984, Geophys. Astrophys. Fluid Dyn. 30, 305

Williams, G.E.: 1981, Nature 291, 624

Williams, G.E.: 1989, J.Geol. Soc. 146, 97

Wilson, O.C.: 1978, Astrophysical J. 226, 379

Yoshimura, H.: 1975, Astrophys. J. Suppl. 29, 467

Yoshimura, H.: 1983, Astrophys. J. Suppl. 52, 363

Zwaan, C.: 1986, in Cool Stars, Stellar Systems and the Sun, M.Zeilik and D.Gibson eds., Springer, Berlin, p.19 